\title{
THE VITAL STAINING OF MUSCLE BIOPSIES WITH METHYLENE BLUE
}

\author{
BY \\ C. COËRS
}

From the Hopital St. Pierre (Clinique Médicale) and the Laboratory of Morbid Anatomy, the University of Brussels

The histological changes of muscle occurring in neuromuscular diseases are often non-specific. They do not always enable us to find out the cause of an atrophy or a degeneration or to assess the part played by a disorder of innervation. Therefore, it would be desirable, when investigating muscle samples taken by biopsy, to be able to study systematically and simultaneously the muscular tissue and the nerve fibres and endings by special staining of axis cylinders and myelin.

The study of biopsies of nerve supply meets two difficulties: (1) the very unequal distribution of nerve endings in various parts of a muscle, so that it is never certain whether a biopsy taken at random will contain any ; (2) the impossibility of getting an idea of the whole innervation of a muscle fragment by silver impregnation methods. Even if a selective and regular impregnation is obtained, and a great number of sections examined, it remains difficult with these methods to decide whether a biopsy contains any nerve endings and fibres, and to learn to recognize their appearance and distribution. Using Holmes' silver on the slide method systematically, I found that the probability of finding some nerve elements in a section of biopsy muscle was very slight. The first step to increase the probability would be to make certain that we obtained neuromuscular biopsies, that is, samples taken from areas of muscle containing abundant nervous elements. Such favourable areas could be mapped out if we were to use a histological method by which the richness in nervous elements of the biopsy tissue could easily be demonstrated. It was with this end in view that $I$ became interested in the use of vital methylene blue.

The classical technique of vital staining in animals consists in perfusing the whole organism with a methylene blue solution, afterwards removing those organs of which the innervation is to be studied (Ehrlich, 1885-86; Dogiel, 1890). Hines and Tower (1928) used this perfusion method in their studies on the innervation of skeletal muscles of the cat. Another procedure is the local injection of the stain, and the subsequent removal of the stained material. Weddell (1941a and b) was able to observe the nerve endings in the skin by this method. I have used a similar method to obtain vital staining of neuromuscular biopsies, and, to the best of my knowledge, this technique of local injection of methylene blue has never been applied to the study of muscular innervation in man. It has proved satisfactory for this purpose, and it is hoped that it may be of some help to those working on muscle biopsies, who have often been discouraged by the difficulty of staining and finding nerve endings in this material.

In this preliminary paper only the technique and the interpretation of the pictures obtained are discussed. However, it is necessary to point out that most of the biopsies upon which this study is based were taken at the " motor point" which was localized, whenever possible, with threshold electrical stimuli. As a rule, the motor point corresponds with the spot where the nerve enters the muscle. It is reasonable to think $a$ priori that there are more chances of finding nerve endings at this place than in an unexcitable part.

\section{Material}

The 31 biopsies at my disposal were taken from various muscles of the superior and inferior limbs : the deltoid, biceps, brachio-radialis, flexor carpi radialis, quadriceps, tibialis anterior, peroneus longus, gastrocnemius; the sterno-cleido-mastoideus, and abducens digiti quinti were each used once.

The patients were affected with either neuromuscular disorders, or primary muscular dystrophies or with systemic diseases in which muscle biopsy might help in diagnosis.

I shall avoid discussing the pictures observed from a physio-pathological point of view, especially since most of the appearances described were 
found in muscles, of which the innervation could be considered normal.

\section{Technique}

After incising the skin and fascia under local anaesthesia with xylocaine without adrenalin, a fragment of muscle is removed for routine biopsy. Then an untouched bundle is selected and treated in the following way. With a thin needle (for intradermal injection) the muscle is infiltrated with an $0.02-0.03 \%$ methylene blue solution, proceeding as for an anaesthetic infiltration in order to produce regular staining. The solution is made up from sterilized $1 \%$ methylene blue diluted in the appropriate amount of normal saline. The stain is allowed to operate for five to 10 minutes, when the stained bundle is removed (about $1 \mathrm{c.cm}$. of muscle tissue) in two or three pieces. It is well known that parenteral methylene blue is harmless. I have never observed any unpleasant reaction following the injection. However, it is to be noticed that the muscle tissue when infiltrated with methylene blue reacts poorly to mechanical stimuli (pinching, cutting).

The samples. moistened with normal saline, are left exposed to the air for from half an hour to one hour. (It seems better to leave them in an atmosphere of pure oxygen.) A darkening of the blue can be seen during this process. The samples are then fixed in a saturated solution of ammonium molybdate for 24 hours at $0^{\circ} \mathrm{C}$., and washed in three changes of distilled water. Further complementary fixation in $10 \%$ neutral formalin takes three to five days. (Too long immersion in formalin may fade the staining of the nerve fibres.)

The fragments are then cut as frozen sections. Before putting them on the stage of the microtome a thin layer of ice is formed on it so that the whole biopsy may be cut without difficulty. Serial sections of 100 to $150 \mu$ are cut. All the sections are kept and mounted on albuminized slides. At this stage it is possible to counterstain the sections with $1 \%$ carmalum.

Finally the sections are immersed in $90 \%$ alcohol, absolute alcohol, and toluol as usual and mounted in a synthetic neutral medium (" harleco" synthetic resin). The staining obtained in this way is permanent. Some sections stained more than a year ago are not at all faded.

\section{Results}

The coloration is usually much more intense at the periphery of the biopsy. This fact is obviously the result of the more complete oxydation of the "leucoderivative" formed in the living tissue. This oxydation occurs only in the surface of the sample when exposed to air. Small bits of tissue have therefore more chance of being fully stained. Using pure oxygen instead, the staining goes deeper and is more regular.

Muscle Fibres.-These are usually stained very pale green or light blue at the periphery of the sections. The sarcolemmal nuclei are never visible, whatever the intensity of the staining. The sarcolemma is sometimes apparent as a thin, wavy blue band, darker than the muscle fibre.

Vascular Endothelia.-These are irregularly stained; usually they are only discernible as an outlining of the capillaries at the periphery of the sections.

Stroma.-Collagen fibres and fat cells are usually little stained. They sometimes show a slight metachromasia. In strongly stained sections the elastic fibres are often well seen. The arteriolar elastic laminae are also well stained.

Nerve Fibres.-These are always the most deeply stained elements. They are always easily recognized amongst the other structures. Even when nothing else is stained, they may often be clearly followed up to their last ramifications. For the axis cylinders as well as for vascular endothelium the oxydation of the section by pure oxygen certainly gives more regular staining.

It is rather uncommon for nerve fibres not to be stained at all. Nevertheless, this staining is far from homogeneous. Sometimes they are uniformly dark blue and their width more or less regular. More often one may observe a dark central filament surrounded by a sheath of irregular width, the staining of which is lighter and irregular. The central filament is frequently interrupted and sometimes replaced by dark blue granulations.

In the less stained preparations, the axis cylinder is uniformly pale blue with a darkening of the stain at the level of Ranvier's nodes where the axis cylinder becomes thinner (Fig. 8). Hence the whole appearance of the nerve fibre is that of a dotted line. Schwann and Henle nuclei are not visible. Myelin sheaths are sometimes apparent and show a metachromatic staining.

Nerve Endings.-My sections showed motor endplates and muscle spindles.

Motor End-plates.-The nuclei of the sole are never visible. The cytoplasm of the sole can sometimes be distinguished from the rest of the muscular fibre by its lighter staining. This difference is more appreciable in deeply stained preparations (Fig. 5). The nervous arborization of the end-plate is easily demonstrated. It stains in a variable manner. As a rule the root of the arborization is deeply stained. One often finds the picture of a central filament surrounded by a paler sheath (Fig. 3). The branches of arborization are thin and less stained, and often end in an easily visible, club-like swelling frequently containing granules (Figs. 1 and 

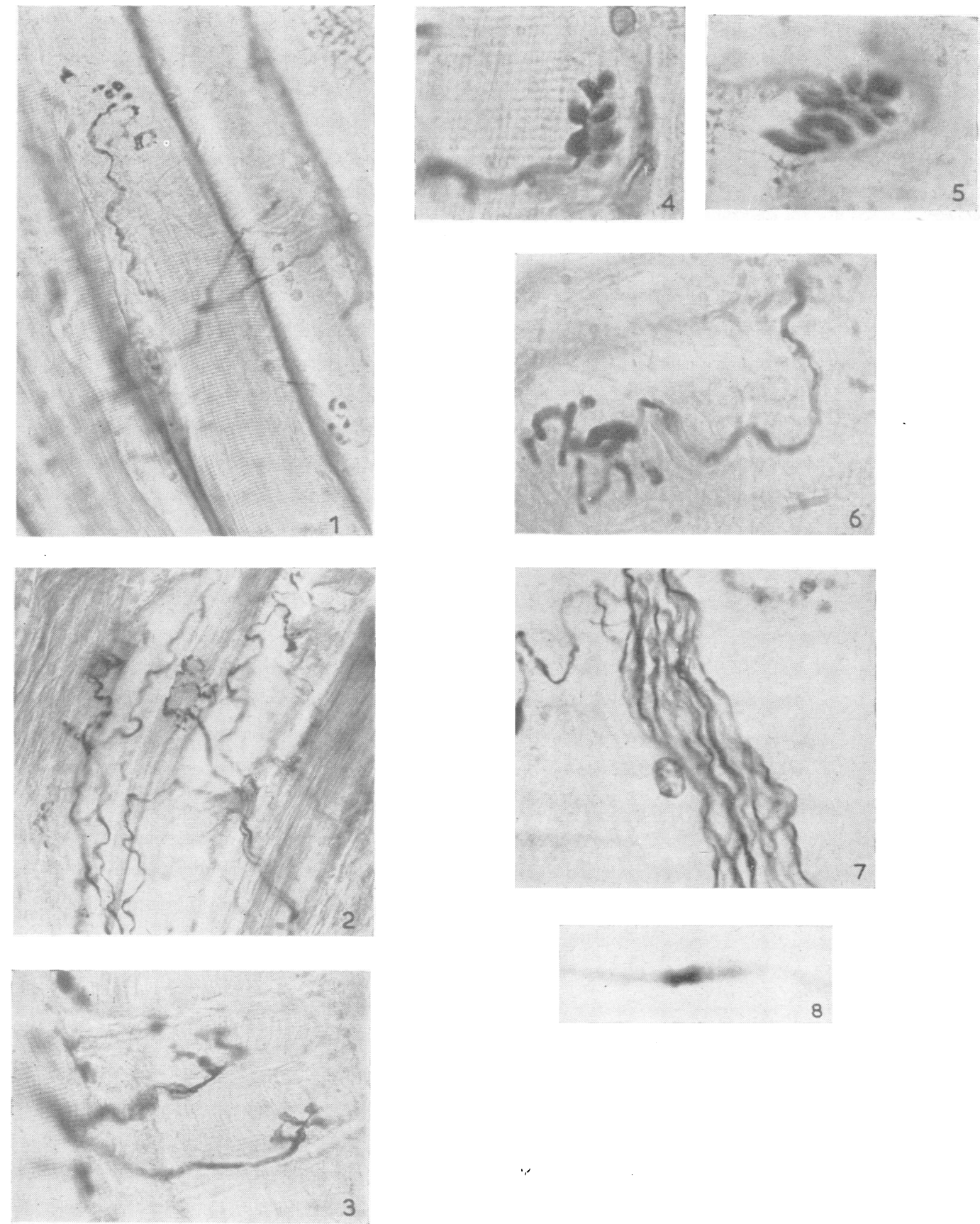

FIG. 1.-Usual aspect of motor end-plates in methylene blue preparation. The upper one is large. It shows the irregular staining of the arborization, the terminal swellings (which are few in this picture) being more deeply stained. At the bottom, a small end-plate is seen, slightly out of focus.

Fig. 2. -This is from a sample fixed in ammonium molybdate only. The transverse striation of the muscle is not visible. The axis cylinders are regularly stained. A triple motor end-plate can be seen.

Fig. 3.-Double motor end-plate. The dark-stained filament surrounded by a paler sheath is well seen on the root of the higher arborization. The granular structure of the endings is noticeable on the lower arborization.

FIG. 4.-Small end-plate resembling a bunch of grapes, because of its well-defined terminal swellings and contracted structure.

Fig. 5.-Strongly stained preparation. Nerve endings are broad. A central filament can be seen. This aspect resembles some of Couteaux's preparations.

Fig. 6.-Large end-plate without terminal enlargements. Compare with Fig. 4.

Fig. 7.-A nerve bundle showing how the axis cylinders usually stain.

Fig. 8.-A slightly stained axis cylinder, showing more intense coloration at Ranvier's narrowing. The narrowing of the axis cylinder can be seen. 

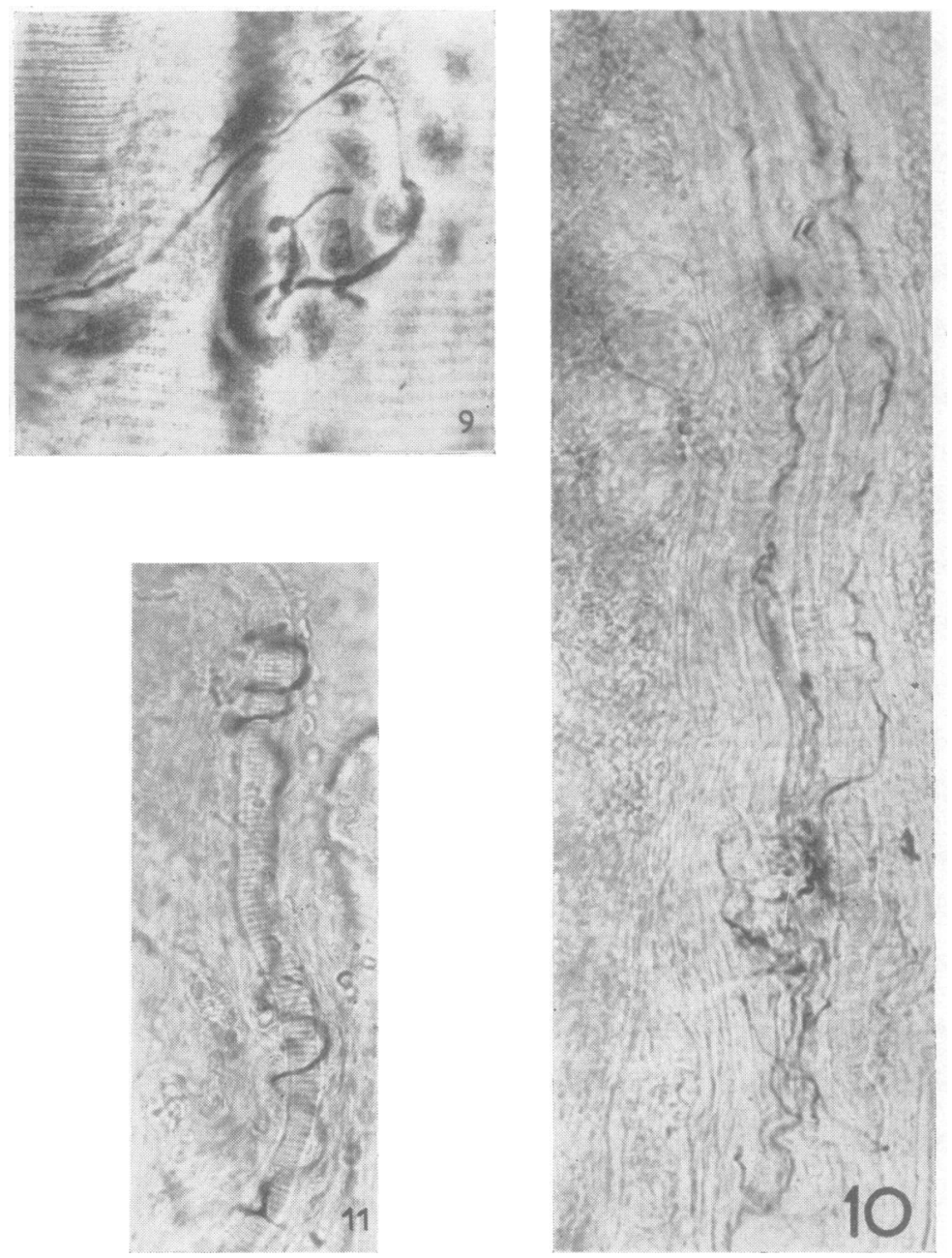

Fig. 9.-Aspect of a motor end-plate in silver impregnation (Holmes method). Compare the staining of the axon with methylene blue preparation.

Fig. 10.-Low magnification of a muscle spindle. Two motor end-plates on intrafusal fibres are visible.

FIg. 11.-High magnification of an intrafusal muscle fibre surrounded by an annulo-spiral nerve fibre.

3). Less often swellings are seen along the course of the fibres. When these terminal swellings arc well formed, the arborizations resemble a bunch of grapes (Fig. 4). Only occasionally the width of the terminal branches remains regular and they do not end in a swelling (Fig. 6). Not only the shape, but also the size of these arborizations is quite variable and may differ in the proportion of 1 to 4 . It must be pointed out that the best formed terminal dilatations are found in the smallest end-plates.

In some preparations I met with a picture that does not correspond with the classical description.
The axis cylinder in the neighbourhood of a muscle fibre divides into two or three branches, each of which ends in a small arborization in the same muscle fibre, thus forming a double (Fig. 3) or triple (Fig. 2) motor end-plate.

Muscle Spindles.-In those parts of muscle rich in fibres and nerve endings, a muscle spindle can not infrequently be found. The nerve endings of these spindles are difficult to stain. They are only seen when the connective sheath has been torn by the needle during injection of the stain. In these more or less damaged spindles, where a haemorrhage is often found, the thin sensory fibres and the motor endings are easy to follow (Fig. 10). The latter are small and show beautiful terminal swellings. The annulospinal fibres and the thick axis cylinders from which they arise are usually pale blue and irregularly stained, and sometimes look like a string of beads.

It seems likely that the methylene blue enters the intact spindle only with difficulty, and that the tearing of the surrounding sheath is a necessary condition for the satisfactory staining of the nerve fibres.

\section{Comments}

Technique.-The optimal concentration of methylene blue appears to be between 0.02 and $0.03 \%$. A higher concentration causes stronger impregnation of the muscle fibres, connective tissue, and elastic fibres, and lessens the contrast with the nerve fibres.

The time of staining of the muscle can be varied to some extent. Diffusion is rapid and the nerve structures are quickly impregnated. I believe that five minutes is long enough. After half an hour the staining of the infiltrated bundle fades and recoloration by exposure to air becomes difficult.

I tried to obtain staining by dipping a fragment of muscle in a dilute solution of methylene blue. The results were unsatisfactory. Only the most superficial parts were strongly and uniformly stained. 
The time of exposure to oxygen is the most important part of the method. During this process the coloration becomes obviously darker. In order to obtain uniform staining, it is wise to treat small fragments of muscle. When the time of exposure to oxygen exceeds an hour, the recoloration becomes too strong. In particular, the vascular endothelia become very dark. Hence the wavy blood capillaries, which anastomose around the muscle fibres, are clearly distinguishable. Also the examination of nerve structures becomes difficult. Thirty to 40 minutes appears to be the optimal time. The action of ammonium molybdate is indispensable for the fixation and preservation of the stain.

I submit the specimens to a further fixation in formalin. This makes the pieces easier to cut, and shows up the muscular striation. In preparations cut after fixation with only ammonium molybdate the transverse striation is not visible (Fig. 2). The formalin tends to cause slight fading of the staining of the axis cylinders. Nevertheless, comparison with preparations untreated with formalin proves that this fixation does not alter the structure of fibres and nerve endings.

\section{Results}

The appearances of muscular nerve endings obtained with methylene blue have been thoroughly described by Dogiel (1890) and Tello (1944). The aspects we observed follow these descriptions fairly closely. They differ slightly from those given by silver impregnations in which the axis cylinder is usually stained homogeneously, and seldom show terminal swellings (cf. Fig. 9). Are these swellings part of the axis cylinder or of the appareil sous-neural (Couteaux, 1947), or do they represent the gaine de l'arborisation (Tello)? What I have been able to observe does not allow me to answer this question as yet. I have never been able to distinguish a sharp demarcation between the axonic thread and these dilatations, even in strongly stained preparations in which the terminal apparatus was particularly wide, and had some analogy with the structures described by Couteaux (Fig. 5).

I have not observed anything resembling Boeke's (1926) " periterminal network". A sharp demarcation always exists between the axis cylinder and the motor end-plate within which it ramifies.

The most interesting observation to be drawn from my preparations is the marked polymorphism of the motor ending. This polymorphism does not appear clearly with silver impregnation, where there is rarely a chance to observe many motor end-plates side by side.

The size and form of the arborizations are most variable : the small motor end-plates usually contain short and thin ramifications ending in large swellings. In the larger end-plates the axonal arborizations are thicker and of more regular calibre; terminal dilatations are less often observed. I found double or triple end-plates, which altogether make up a very large nerve ending. It is tempting to compare these structural differences with the appearances described by Carey (1941), but it is not my intention to discuss their functional significance as this author has done.

\section{Conclusion}

Vital staining with methylene blue can be used on biopsies of muscle. It easily gives results which are fairly reliable. This method is therefore of value for the evaluation of the richness in nervous elements of a muscle sample, and it may be used to map out the muscular territories which have the richest innervation.

The most interesting application of this method seems to be in studies on neuromuscular pathology.

\section{Summary}

A method of vital staining of muscle biopsies with methylene blue is described. A dilute solution of the stain is injected into the muscle to be examined. After removal, the sample is exposed to oxygen, then fixed in ammonium molybdate. After further fixation in formalin, the biopsy is cut in thick frozen sections, which are mounted in the usual way.

The results are described and their value discussed.

The polymorphism of motor end-plates shown by this method is emphasized.

I wish to thank Professor P. Gerard, in whose laboratory this work was performed, and Dr. J. G. Greenfield, who kindly helped me with the English translation and preparation of this paper.

\section{REFERENCES}

Boeke, J. (1926). Z. mikr. anat. Forsch., 4, 448.

Carey, E. J. (1941). Anat.-Rec., 81, 393.

Couteaux, R. (1947). Contribution à l'étude de la synapse myoneurale. Thèse de Paris. Thérien. Montreal.

Dogiel, A. S. (1890). Arch. mikr. Anat., 35, 305.

Ehrlich, P. (1886). Dtsch. med. Wschr., No. 4.

Hines, M., and Tower, S. S. (1928). Bull. Johns Hopk. Hosp., Hines, 264.

Tello, J. F. (1944). Trab. Inst. Cajal Invest. biol., 36, 1.

Weddell, G. (1941a). J. Anat., Lond., 75, 346.

-(1941b). Ibid., 75, 441 . 